\title{
Vibrational stabilization of ultracold KRb molecules. A comparative study
}

\author{
Mamadou Ndong and Christiane P. Koch $*$ \\ Institut für Theoretische Physik, Freie Universität Berlin, Arnimallee 14, 14195 Berlin, Germany
}

(Dated: November 21, 2018)

\begin{abstract}
The transfer of weakly bound KRb molecules from levels just below the dissociation threshold into the vibrational ground state with shaped laser pulses is studied. Optimal control theory is employed to calculate the pulses. The complexity of modelling the molecular structure is successively increased in order to study the effects of the long-range behavior of the excited state potential, resonant spinorbit coupling and singlet-triplet mixing.
\end{abstract}

PACS numbers: 33.80.-b,32.80.Qk,82.53.Kp

\section{INTRODUCTION}

Research on cold and ultracold molecules has been one of the most active areas of atomic and molecular physics over the last decade and continues to draw much attention [1]. Current activities are by and large still focussed on producing (ultra)cold molecules, either by direct cooling or by assembling molecules from cooled atoms using external fields. While direct methods such as Stark deceleration [2] have not yet reached the regime of ultracold temperatures $(T \leq 100 \mu \mathrm{K})$, photo- and magnetoassociation create molecules in their electronic ground state that are ultracold but in highly excited vibrational levels [3-5]. However, prospective applications ranging from high-precision measurements to quantum information carriers [1] require stable ultracold molecules. This has triggered the quest for molecules in their absolute ground state. A major breakthrough toward this longstanding goal was achieved when several groups produced molecules in the lowest rovibrational level of an electronic ground state potential via Stimulated Raman Adiabatic passage [6, 7], photoassociation [8, 9] and vibrational laser cooling 10]. Finally, the ability to control not only the rovibrational but also the hyperfine degree of freedom has recently paved the way toward ultracold molecules in their absolute ground state [11].

An earlier, alternative proposal to reach molecules in their vibronic ground state was based on employing laser pulse shaping capabilities: An optimally shaped laser pulse can coherently transfer, via many Raman transitions, vibrationally highly excited molecules into $v=0$ [12]. The attractiveness of this proposal rests on the fact that optimization is carried out iteratively, both in experiment and in theory, and does not require detailed knowledge of the molecular structure to identify optimal pulses. One might nevertheless ask whether and how qualitative changes in the molecular structure affect the optimal solution. This question defines the scope of the present work. We solve the same optimization problem - population transfer from a weakly bound vibrational level just below the dissociation threshold of the

*Electronic address: christiane.koch@physik.fu-berlin.de electronic ground state potential to $v=0$ - for two different molecules, $\mathrm{KRb}$ and $\mathrm{Na}_{2}$, successively taking effects into account that strongly alter the molecular structure of KRb. Our choice to focus on this molecule is motivated by the long-standing [6, 13-18] and continuing [11, 19, 20] experimental efforts on KRb.

The paper is organized as follows: The general theoretical approach is described in Section [II Sections [II.V present and analyze the short shaped laser pulses that achieve the vibrational transfer. The complexity of the model for the molecular structure of KRb is successively increased, cf. Fig. 1. Based on a two-state model, Section III compares the optimization of vibrational transfer for $\mathrm{KRb}$ and $\mathrm{Na}_{2}$ molecules. They differ in the longrange behavior of their excited state potentials, $1 / R^{3}$ vs $1 / R^{6}$. Section IV is dedicated to the effect of spinorbit interaction in the electronically excited state which may lead to resonant coupling between a singlet and a triplet state 21 23]. This is investigated with a threestate model in Section IV. Adding one more channel, Section $\mathrm{V}$ studies the transfer of a coherent superposition of singlet and triplet molecules in their electronic ground state into the ground vibrational level of the singlet potential. This is possible for polar molecules due to the broken gerade-ungerade symmetry [8]. Section VI concludes.

\section{THEORETICAL APPROACH}

\section{A. Model}

The linear Schrödinger equation describing the internuclear dynamics of two atoms is considered. For molecules formed in a quantum degenerate gas, the many-body dynamics are then neglected. This approach is justified by the time scales of standard optical and/or magnetic traps 24]. While the internuclear dynamics and pulse shaping occur on the time scale of picoseconds, the many-body dynamics for conventional traps is characterized by microseconds. The many-body system will have to adjust to the new internal state, but this is going to happen on a much slower time scale only after the pulse is over [25]. 

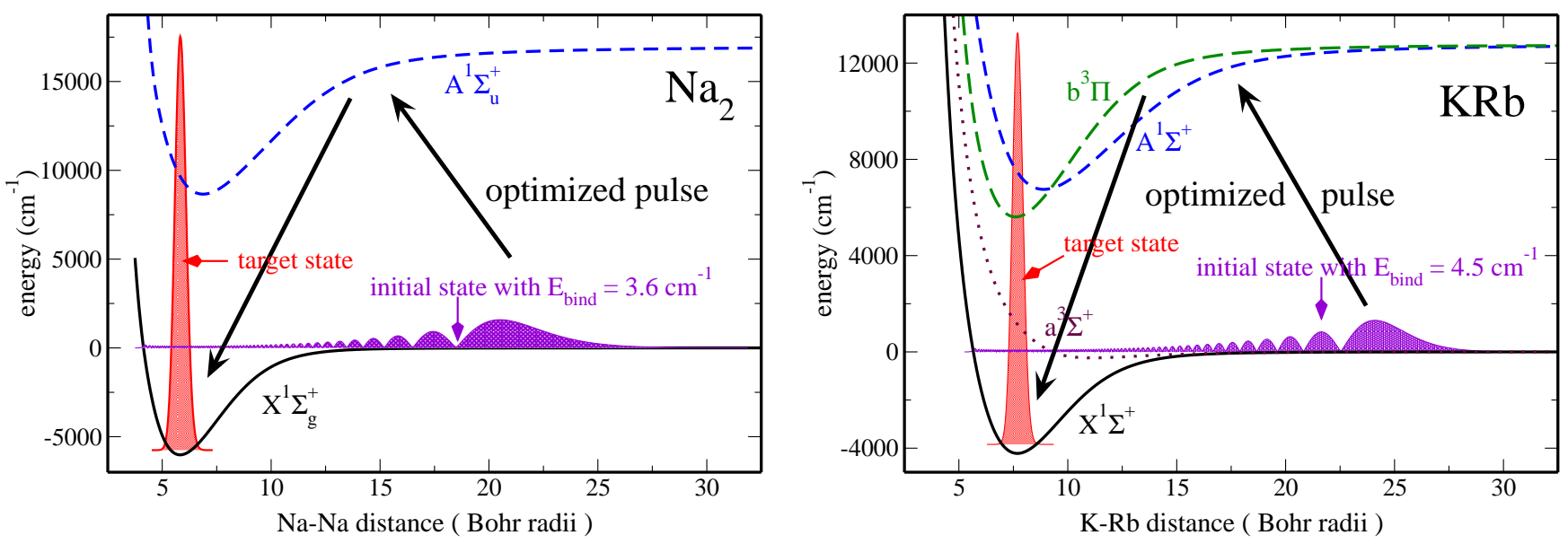

FIG. 1: (color online) Potential energy curves and initial and target wave functions for vibrational stabilization of Na2 (left) and $\mathrm{KRb}$ (right) molecules. The complexity of the model for the molecular structure of KRb is successively increased from two to four channels.

The time-dependent Schrödinger equation,

$$
i \frac{\partial}{\partial t}|\psi(t)\rangle=\left(\hat{\mathbf{H}}_{0}+\hat{\boldsymbol{\mu}} \varepsilon(t)\right)|\psi(t)\rangle
$$

is solved with a Chebychev propagator [26]. The Hamiltonian comprises two or more electronic states as specified below, cf. Fig. 1. The interaction with the laser field is treated in the dipole approximation. The rotating wave approximation is not invoked in order to allow for strong fields and multi-photon transitions. The Hamiltonian and the wavefunctions, $\psi_{i}(R)=\left\langle R \mid \psi_{i}\right\rangle$, where $i$ denotes the channel, are represented on a Fourier grid with adaptive grid step [27-29]. In order to obtain vibrational eigenfunctions and Franck-Condon factors, the Hamiltonian, $\hat{\mathbf{H}}_{0}$, is diagonalized.

\section{B. Optimal control theory}

Denoting the formal solution of the Schrödinger equation at time $t$ by

$$
|\psi(t)\rangle=\hat{\mathbf{U}}(t, 0)|\psi(0)\rangle,
$$

the objective functional for a transition from initial state $\left|\psi_{\text {ini }}\right\rangle$ to target state $\left|\psi_{\text {target }}\right\rangle$ at the final time $T$ can be written

$$
F=\left|\left\langle\psi_{\text {ini }}\left|\hat{\mathbf{U}}^{\dagger}(T, 0 ; \varepsilon)\right| \psi_{\text {target }}\right\rangle\right|^{2} .
$$

It corresponds to the overlap of the initial state that has been propagated to time $T$ under the action of the field $\varepsilon(t)$ with the target state. A field is optimal if it completely transfers the initial state, $\left|\psi_{\text {ini }}\right\rangle$, to the target state, $\left|\psi_{\text {target }}\right\rangle$, i.e. if $F$ reaches a value close to one.

The objective $F$ is a functional of the field $\varepsilon(t)$. It explicitly depends only on the final time $T$. In order to use dynamical information from intermediate times, a new functional is defined,

$$
J=-F+\int_{0}^{T} g(\varepsilon) d t
$$

where $g(\varepsilon)$ denotes an additional constraint over the field. Often $g(\varepsilon)$ is chosen to minimize the pulse fluence. This implies a replacement rule in the control equation for the field. However, a choice of $g(\varepsilon)$ that leads to vanishing changes in the field as the optimum is reached may be advantageous from the point of view of convergence [30]. It is employed here,

$$
g(\varepsilon)=\frac{\lambda}{S(t)}[\varepsilon(t)-\tilde{\varepsilon}(t)]^{2},
$$

and $\tilde{\varepsilon}(t)$ is taken to be the field of the previous iteration. This choice of $g(\varepsilon)$ implies an update rule rather than a replacement rule in the control equation for the field. Physically, it corresponds to minimizing the change in pulse energy at each iteration. The shape function $S(t)$, $S(t)=\sin ^{2}(\pi t / T)$, enforces a smooth switch-on and off of the field. The parameter $\lambda$ controls the optimization strategy: A small value results in a small weight of the constraint, Eq. (5), allowing for large modifications in the field, while a large value of $\lambda$ represents a conservative search strategy with only small modifications in the field at each iteration.

We employ the Krotov algorithm [30 34 to obtain the control equations. The derivation of the algorithm for the target, Eq. (3), and the constraint, Eq. (5), is described in detail in Ref. [12]. It yields the following prescription to improve the field, 


$$
\varepsilon_{j}(t)=\varepsilon_{j-1}(t)+\frac{S(t)}{\lambda} \mathfrak{I m}\left\{\left\langle\psi_{\text {ini } i}\left|\hat{\mathbf{U}}^{\dagger}\left(T, 0 ; \varepsilon_{j-1}\right)\right| \psi_{\text {target }}\right\rangle\left\langle\psi_{\text {target }}\left|\hat{\mathbf{U}}^{\dagger}\left(t, T ; \varepsilon_{j-1}\right) \hat{\boldsymbol{\mu}} \hat{\mathbf{U}}\left(t, 0 ; \varepsilon_{j}\right)\right| \psi_{\text {ini }}\right\rangle\right\}
$$

at the $j$ th iteration step. The first overlap in the parenthesis can be shown to be time-independent. The second overlap contains a backward propagation of the target state from time $T$ to time $t$ under the old field, $\varepsilon_{j-1}(t)$, and a forward propagation of the initial state under the new field, $\varepsilon_{j}(t)$. Eq. (6) is implicit in $\varepsilon_{j}(t)$. This is remedied by employing two different grids in the time discretization, see Ref. [12] for details.

\section{THE ROLE OF THE LONG-RANGE BEHAVIOR OF THE EXCITED STATE POTENTIAL: COMPARING KRB TO NA${ }_{2}$}

The investigation is started with a simple two-state model. The Hamiltonian reads

$$
\hat{\mathbf{H}}_{2 s}(t)=\left(\begin{array}{cc}
\hat{\mathbf{T}}+V_{X^{1} \Sigma_{(g)}^{+}}(\hat{\mathbf{R}}) & \hat{\boldsymbol{\mu}} \varepsilon(t) \\
\hat{\boldsymbol{\mu}} \varepsilon^{*}(t) & \hat{\mathbf{T}}+V_{A^{1} \Sigma_{(u)}^{+}}(\hat{\mathbf{R}})
\end{array}\right),
$$

where $(g / u)$ applies only to $\mathrm{Na}_{2}$. $\hat{\mathbf{T}}$ denotes the kinetic energy operator and $V_{i}(\hat{\mathbf{R}})$ the potential energy curve of channel $i$. For the $\mathrm{Na}_{2}$ molecule, we employ the same potential energy curves as in Ref. [12 54 which were obtained from molecular spectroscopy [35, 36]. The ground state potential for KRb is also known spectroscopically [37]. The excited state potential energy curves of $\mathrm{KRb}$ are unfortunately not yet known with spectroscopic precision. We have therefore employed the results of $a b$ initio calculations [38] at short internuclear distances together with an asymptotic expansion of the form $V_{\text {asy }}(R)=-\frac{C_{6}}{R^{6}}-\frac{C_{8}}{R^{8}}$ with the long-range coefficients taken from Ref. [39]. The laser field, $\varepsilon(t)$, couples to the molecule via the transition dipole moment, $\hat{\boldsymbol{\mu}}$. The latter is expected to depend on internuclear distance at least for short $R$. However, it is approximated by $\hat{\boldsymbol{\mu}}=1 \mathrm{a} . \mathrm{u}$. since no data on the $R$-dependence could be found in the literature.

The initial state, $\psi_{\text {ini }}(R)=\left\langle R \mid \psi_{\text {ini }}\right\rangle=\psi_{g}^{v}(R)$ is taken to be a vibrational wavefunction of the electronic ground state corresponding to a weakly bound level just below threshold. Counting the levels downward from the last bound one, $v=v_{\text {last }}-3$ for $\mathrm{Na}_{2}$ and $v=v_{\text {last }}-6$ for $\mathrm{KRb}$. These levels were chosen to yield a comparable binding energy for the two species. They are very similar to levels that would be populated when molecules are formed from atoms using a Feshbach resonance or photoassociation but with a somewhat larger binding energy. This approximation eases calculations since, as discussed below, the absolute value of the optimization time is determined by the binding energy of the initial state. It does not affect the comparison between $\mathrm{Na}_{2}$ and $\mathrm{KRb}$ which is based on relative times.

While the model, Eq. (7), does not capture the full complexity of the molecular structure, it allows for a straightforward comparison with earlier work on the $\mathrm{Na}_{2}$ molecule [12]. In particular, it is useful to highlight the influence of the long-range behavior of the excited state potential which is $1 / R^{6}$ for $\mathrm{KRb}$ but $1 / R^{3}$ for $\mathrm{Na}_{2}$. From a spectroscopic point of view, one expects the $1 / R^{6}$ behavior of KRb to be more favorable for vibronic transitions between highly excited vibrational levels. This is attributed to better Franck-Condon overlaps between alike potentials. However, from a dynamical point of view, a maximum difference potential between electronic ground and excited state, $\Delta V(R)=\frac{1}{2}\left(V_{e}(R)-V_{g}(R)\right)$, is more desirable. A larger difference potential is obtained for an excited state with $1 / R^{3}$ long-range behavior, i.e. for $\mathrm{Na}_{2}$. The reasoning behind this argument is that the wave packet that launched by the pulse on the excited state experiences a much larger gradient and can better accelerate its motion toward shorter distances [40].

In order to decide which of the two arguments is relevant for vibrational stabilization of ultracold molecules, we determine the minimum pulse energy and minimum optimization time required to achieve a transfer to the target state of $99 \%$ or better. This is based on the fact that many solutions to the control problem exist. Which solution will be found by the algorithm depends crucially on the boundary conditions - optimization time and pulse power or pulse energy. However, if the resources in terms of time and energy are insufficient, no solution will be found. The lower limits to $T$ and $\mathcal{E}_{P}$ can thus be used to characterize the control problem and the solution strategy.

Figure 2 compares the optimal pulses and their spectra for $\mathrm{Na}_{2}$ (left) and $\mathrm{KRb}$ (right). These pulses are the results of a three-step optimization. Initially, an optimization time of $T=16 \mathrm{ps}$ corresponding to about twice the vibrational period of the initial state of $\mathrm{Na}_{2}$ was chosen [12]. The guess pulses for this optimization were constructed as a series of $100 \mathrm{fs}$ pulses with two central frequencies reflecting the peaks of the Franck-Condon factors of the initial and the target states. This choice provides a large enough spectral bandwidth for given $T$. The pulses were optimized until a transfer of $99 \%$ or better was achieved. For the second optimization step, the pulses were compressed in time following the recipe detailed in Ref. [12], i.e. points $\varepsilon\left(\omega_{i}\right)$ were removed such that $\Delta \omega$ is increased and $T$ decreased. The resulting pulses were then employed as guess pulses for the second step of optimization. For $\mathrm{Na}_{2}$, compression of $T$ by a factor of 8 was possible while for KRb a factor of 4 

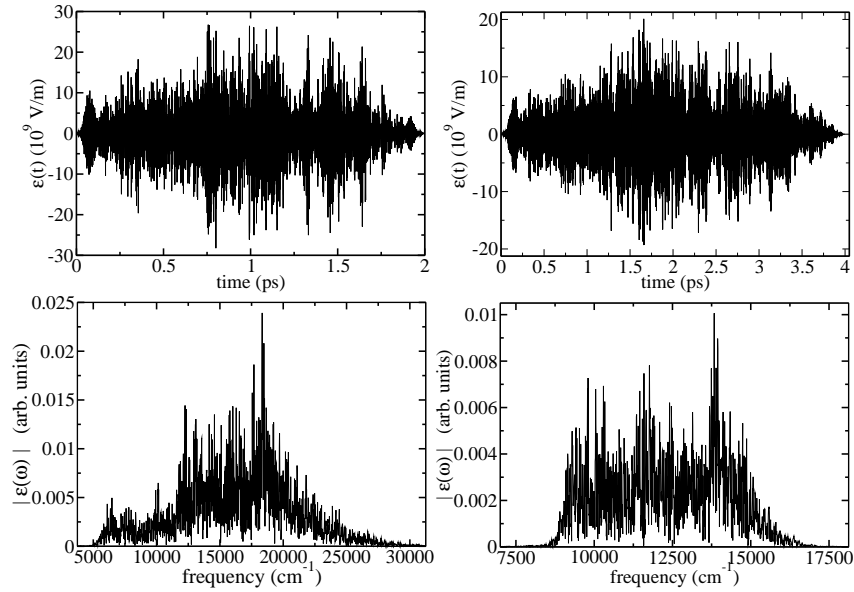

FIG. 2: Optimal pulses (upper panel) and their spectra (lower panel) for $\mathrm{Na}_{2}$ (left) and $\mathrm{KRb}$ (right).

turned out to be the limit. For larger compression factors, the optimization did not result in any appreciable population transfer to the target state. The difference in the minimum optimization time $T$ for $\mathrm{Na}_{2}$ and $\mathrm{KRb}$ is explained in terms of the different reduced masses of the molecules. Although the binding energy of the initial states is comparable, cf. Fig. 1, the motion of the heavier $\mathrm{KRb}$ is slower. This is reflected in the vibrational period of the initial states, $8 \mathrm{ps}$ for $\mathrm{Na}_{2}$ compared to $14 \mathrm{ps}$ for $\mathrm{KRb}$. Therefore, the compression factor taken with respect to the corresponding vibrational period, is 4 for $\mathrm{Na}_{2}$ compared to 3.5 for $\mathrm{KRb}$, i.e. the maximum factor for compression in time is very similar. Finally, the minimum energy required for optimal transfer was determined in step three where the guess pulses were taken to be the optimal pulse of the previous step divided in amplitude by some factor. If the factor was too large, optimization did not result in any appreciable population transfer, otherwise $99 \%$ transfer or better were achieved. This way, a sharp limit for the mininum required pulse energy was obtained. It amounts to $78 \mu \mathrm{J}$ for $\mathrm{Na}_{2}$ and $61 \mu \mathrm{J}$ for KRb where the focal radius of the laser beam is assumed to be $100 \mu \mathrm{m}$.

The lower bounds on the optimization time and the pulse energy represent the main difference between the optimal pulses for $\mathrm{Na}_{2}$ and KRb. As can be seen in Fig. 2, the overall temporal and spectral structures of the pulses are fairly similar. The spectral bandwidth of the optimal pulse is significantly larger for $\mathrm{Na}_{2}$ than for KRb. It is, however, difficult to attach a physical meaning to the spectral bandwidth. We do not filter out undesired spectral components [41, 42], i.e. those components that do not correspond to given vibronic transitions in order to avoid significantly increased convergence times and numerical effort. A constraint formulated directly in frequency domain to contain the bandwidth within a certain spectral region cannot be enforced simultaneously with Eq. (5) [12]. Therefore, with progressing iteration, the spectral bandwidth of the optimized pulses grows.
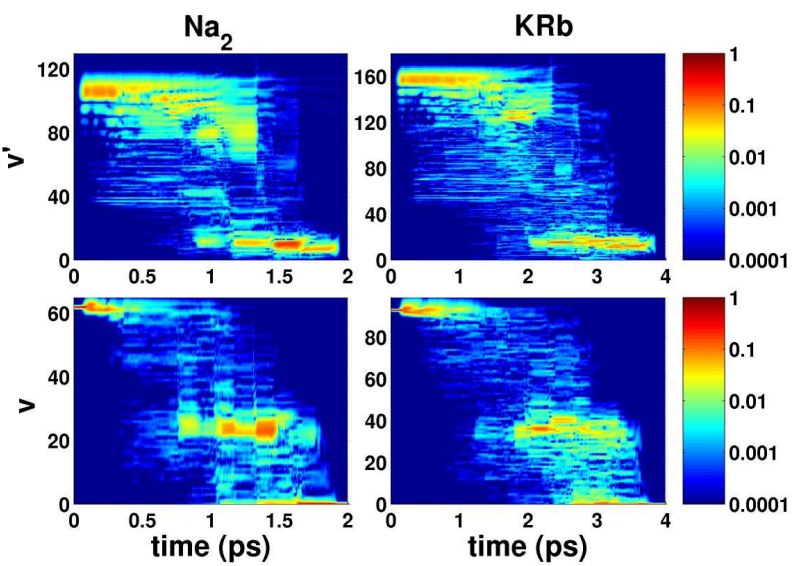

FIG. 3: (color online) Projection of the time-dependent wave packets onto the vibrational eigenstates of the electronic ground (bottom) and excited (top) states for $\mathrm{Na}_{2}$ (left) and $\mathrm{KRb}$ (right)
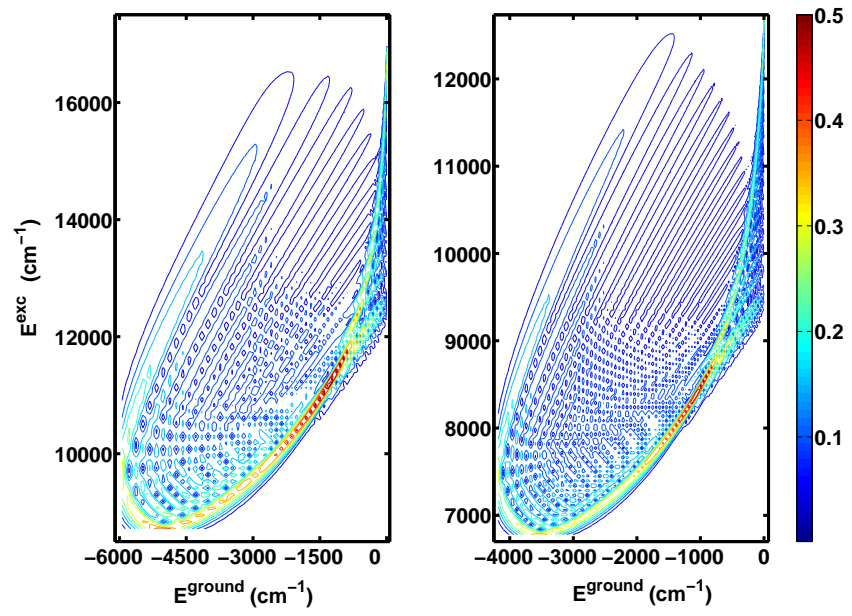

FIG. 4: (color online) Franck-Condon factors of the ground state levels with all excited state levels for $\mathrm{Na}_{2}$ (left) and KRb (right). The dissociation limit of the electronic ground state defines the zero of energy and the dissociation limit of the excited state is $16965 \mathrm{~cm}^{-1}$ for $\mathrm{Na}_{2}$ and $12737 \mathrm{~cm}^{-1}$ for $\mathrm{KRb}$.

Since in principle this growth can be suppressed by filtering [41, 42], it is rather an artifact of the algorithm than a physically significant finding.

The dynamics induced by the optimal pulses are analysed in Fig. 3 by projecting the ground and excited state components of the time-dependent wave packets onto the vibrational eigenstates. The dynamics show similar features for $\mathrm{Na}_{2}$ and $\mathrm{KRb}$. The optimal pulse depletes the initial state and pumps most of the population to the electronically excited state, distributing it over a wide range of highly excited vibrational levels. The wave packet then climbs down the potentials. In the middle of the optimization time interval, it reaches ground state levels $v \approx 30(v \approx 40)$ for $\mathrm{Na}_{2}(\mathrm{KRb})$ ground state which 
is about half way down to the bottom of the potential well. In the last quarter of the optimization time interval, population is accumulated in a superposition of a few excited state levels around $v^{\prime} \approx 10$ for $\mathrm{Na}_{2}\left(v^{\prime} \approx 18\right.$ for $\mathrm{KRb})$. In a final step, this superposition is transfered to the target level, $v=0$.

The population dynamics is rationalized by an analysis of the Franck-Condon map, cf. Fig. 4, The dynamics start in the upper right corner of the map and follow the main ridge until $v \approx 30(v \approx 40)$ for $\mathrm{Na}_{2}$ (KRb) is reached. At this point the Franck-Condon map takes approximately the shape of a parabola where the right branch connects to $v \approx 30(v \approx 40) \mathrm{Na}_{2}(\mathrm{KRb})$ while the left branch connects to the target level, $v=0$. This explains the dynamics in the second half of the optimization time interval where the population is pumped into the excited state levels, $v^{\prime} \approx 10$ for $\mathrm{Na}_{2}\left(v^{\prime} \approx 18\right.$ for $\left.\mathrm{KRb}\right)$, that are reached by the two branches of the parabola, i.e. that are the ideal gateway to $v=0$.

To conclude the comparison of $\mathrm{Na}_{2}$ and $\mathrm{KRb}$, the min- imum optimization time is dictated by the mass of the molecule and population transfer in terms of required pulse energy is more favorable for a $1 / R^{6}$ than a $1 / R^{3}$ excited state potential. The overall dynamics of the population transfer is rather similar for the two molecules and is easily rationalized by the structure of the FranckCondon map.

\section{SPIN-ORBIT COUPLING IN THE ELECTRONICALLY EXCITED STATE}

The complexity of our model is increased to take spinorbit interaction in the electronically excited state of $\mathrm{KRb}$ into account. Similarly to the $\mathrm{Rb}_{2}$ and $\mathrm{RbCs}$ molecules, spin-orbit interaction may lead to resonant coupling and strong non-adiabatic effects [21 23]. This is captured by a three-state Hamiltonian,

$$
\hat{\mathbf{H}}_{3 s}(t)=\left(\begin{array}{ccc}
\hat{\mathbf{T}}+V_{X^{1} \Sigma^{+}}(\hat{\mathbf{R}}) & \hat{\boldsymbol{\mu}} \varepsilon(t) & 0 \\
\hat{\boldsymbol{\mu}} \varepsilon^{*}(t) & \hat{\mathbf{T}}+V_{A^{1} \Sigma^{+}}(\hat{\mathbf{R}}) & W_{S O}^{\Sigma \Pi}(\hat{\mathbf{R}}) \\
0 & W_{S O}^{\Sigma \Pi}(\hat{\mathbf{R}}) & \hat{\mathbf{T}}+V_{b^{3} \Pi}(\hat{\mathbf{R}})-W_{S O}^{\Pi \Pi}(\hat{\mathbf{R}})
\end{array}\right)
$$

where the diagonal and off-diagonal spin-orbit interaction terms, $W_{S O}^{\Pi \Pi}(\hat{\mathbf{R}})$ and $W_{S O}^{\sum \Pi}(\hat{\mathbf{R}})$, are introduced. In principle, the $b^{3} \Pi$ excited state has a dipole coupling with the lowest triplet state. One can thus transfer molecules from the lowest triplet state via Raman transitions into the singlet ground state. This will be investigated below in Section V, and the present section is devoted to studying the effect of non-adiabaticities in the electronically excited state. Here, the initial state is purely singlet, i.e. the same weakly vibrational level of the $X^{1} \Sigma^{+}$ state, $v=93=v_{\text {last }}-6$, as in the previous section is considered.

Unlike in the case of $\mathrm{Rb}_{2}$ where the spin-orbit interaction terms were determined spectroscopically [43], no such accurate data is available for $\mathrm{KRb}$. We have therefore resorted to the parametrization of $W_{S O}^{\Pi \Pi}(\hat{\mathbf{R}})$ and $W_{S O}^{\Sigma \Pi}(\hat{\mathbf{R}})$ in terms of Morse functions,

$$
\begin{gathered}
W_{S O}^{j}(\hat{\mathbf{R}})=P_{1}^{j}+\left(P_{2}^{j}-P_{1}^{j}\right)\left(1-e^{P_{3}^{j}\left(P_{4}^{j}-\hat{\mathbf{R}}\right)}\right)^{2}, \\
j=\Pi \Pi, \Sigma \Pi
\end{gathered}
$$

that was introduced by Bergeman et al. for RbCs [44]. These two functions show a dip at intermediate distances and level off toward a constant value at long range. As a first guess, we have employed the values for the parameters $P_{i}^{j}$ from Ref. [44] scaled to reproduce the correct asymptotic limit of the fine-structure splitting of $237.595 \mathrm{~cm}^{-1}$. The corresponding values for the param- eters $P_{i}^{j}$ are listed in the first row of Table I. Since the parameters $P_{i}^{j}$ are not accurately known, we have varied the $P_{i}^{j}$ in order to estimate the maximum effect that the spin-orbit interaction can have on the vibrational wave functions and Franck-Condon matrix elements. This provides the starting point for studying the strongest possible effect of the spin-orbit interaction on the optimization and the dynamics under the optimal pulse. Two different choices of spin-orbit coupling are employed, referred to below as cases 1 and 2. The corresponding parameters are listed in the second and third rows of Table 1 . The modification of the parameters is quite substantial and larger than what can realistically be expected. However, the point here is to demonstrate the most positive and most negative effect that the spin-orbit coupling may have on the vibrational stabilization and to explore its influence on the optimization.

In case 1 , we have modified $P_{1}^{\Pi \Pi}$ and $P_{3}^{\Sigma \Pi}$. The latter corresponds to the width of the dip in the off-diagonal spin-orbit coupling, while the former represents the constant offset of the diagonal spin-orbit coupling, which essentially causes a relative shift of the vibrational ladders of the $A^{1} \Sigma$ and $b^{3} \Pi$ states. This choice of parameters leads to strong resonant coupling and strongly perturbed vibrational wavefunctions where each diabatic component shows peaks at the four classical turning points of both potentials. As illustrated in the middle panel of Fig.5, such a situation is potentially favorable for vibra- 


\begin{tabular}{|c|c|c|c|c|c|c|c|c|c|c|}
\hline & $\mathrm{j}$ & $P_{1}^{j}\left(\mathrm{~cm}^{-1}\right)$ & $P_{2}^{j}\left(\mathrm{~cm}^{-1}\right)$ & $P_{3}^{j}\left(\AA^{-1}\right)$ & $P_{4}^{j}(\AA)$ & $\mathrm{j}$ & $P_{1}^{j}\left(\mathrm{~cm}^{-1}\right)$ & $P_{2}^{j}\left(\mathrm{~cm}^{-1}\right)$ & $P_{3}^{j}\left(\AA^{-1}\right)$ & $P_{4}^{j}(\AA)$ \\
\hline RbCs [44], scaled & $\Pi \Pi$ & 135.49 & 184.70 & 0.24 & 5.82 & $\Sigma \Pi$ & 130.77 & 261.20 & 0.23 & 5.85 \\
\hline case 1 & $\Pi \Pi$ & 180.49 & 184.70 & 0.24 & 5.82 & $\Sigma \Pi$ & 130.77 & 261.20 & 0.5 & 5.85 \\
\hline case 2 & $\Pi \Pi$ & 135.49 & 184.70 & 0.24 & 5.82 & $\Sigma \Pi$ & 130.77 & 261.20 & 0.5 & 5.85 \\
\hline
\end{tabular}

TABLE I: Parameters of the spin-orbit coupling functions, cf. Eq. (9).

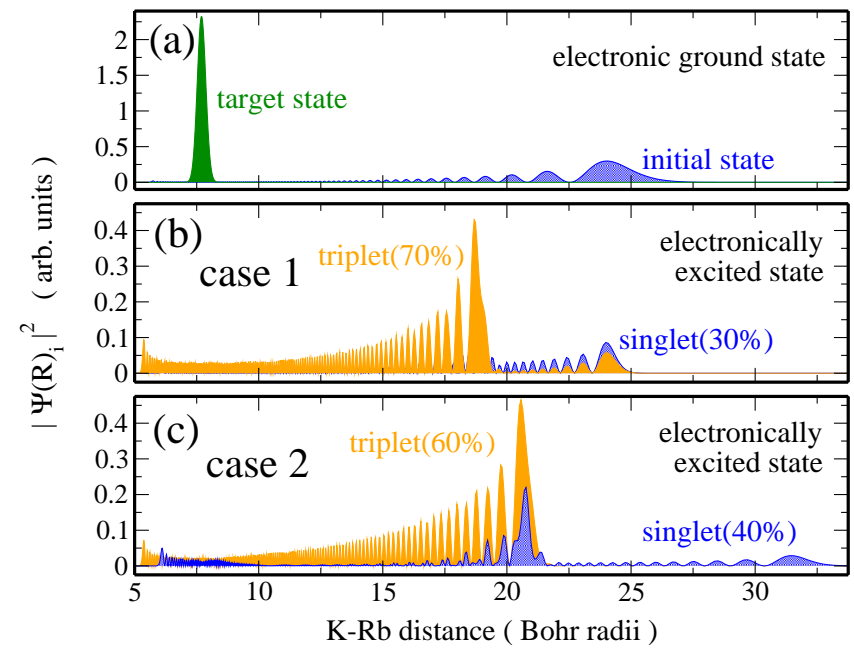

FIG. 5: (color online) Initial and target vibrational wavefunctions of the electronic ground state (upper panel) and effect of the spin-orbit coupling on the vibrational wavefunctions of the electronically excited states (medium and bottom panel).

tional stabilization: The outermost peak of the vibrational wavefunction with a binding energy of $112 \mathrm{~cm}^{-1}$ (corresponding to an absolute energy of $12625 \mathrm{~cm}^{-1}$ ) leads to good Franck-Condon overlap with the initial state. The singlet component of the wavefunction shows a second peak at the outer turning of the $b^{3} \Pi$ state, $R \approx 19 \mathrm{a}_{0}$. This second peak will lead to better overlap with more deeply bound levels in the electronic ground state and could thus cause a speed-up of the stabilization dynamics toward shorter distances or less required pulse energy. Note that the Franck-Condon overlap reflects only the singlet component of the vibrational wavefunctions. However, due to the time-dependence of the stabilization process, the triplet component may play a role as well. If there is a dynamical interplay of pulse and spinorbit coupling, the transfer efficiency can be much larger than predicted by static Franck-Condon overlaps [45]. Such a situation occurs for strong pulses and pulse durations comparable to or longer than the period of the singlet-triplet oscillations caused by the spin-orbit interaction. Both conditions will be met by the optimized pulses presented below.

In case 2 , we have only modified the width of the dip in the off-diagonal spin-orbit coupling, $P_{3}^{\Sigma \Pi}$. As in case 1 , strong perturbations in the vibrational wavefunctions are observed, cf. the peaks at the two outer turning points in the singlet component in the lower panel of Fig. 5. How-
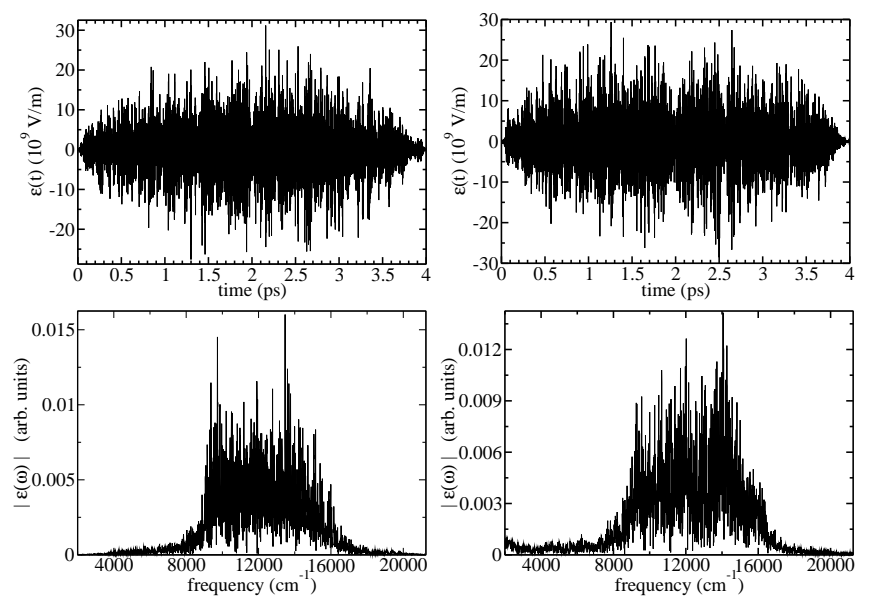

FIG. 6: Optimal pulses (upper panel) and their spectra (lower panel) for the spin-orbit coupling cases 1 (left) and 2 (right).

ever, the spin-orbit coupling is now expected to have a detrimental effect on vibrational stabilization where the wave packet shall be transferred from large to short distances: Once the wavepacket comes close to the outer turning point of the upper adiabatic potential, $R \approx 21 \mathrm{a}_{0}$, the resonant spin-orbit coupling will move part of the probability amplitude all the way out to the outer turning point of the lower adiabatic potential, $R \approx 32 \mathrm{a}_{0}$. Therefore in case 2 , the spin-orbit coupling will potentially counteract the vibrational stabilization.

The same three-step optimization procedure as in Section III has been followed: (i) optimization for $T=16 \mathrm{ps}$, (ii) compression in time to $T=4 \mathrm{ps}$ and subsequent re-optimization, (iii) determination of the minimal integrated pulse energy with which a population transfer of better than $99 \%$ can be achieved. However, the guess pulses for step (i) were chosen such as to take the modified Franck-Condon factors into account. Figure 6] compares the optimal pulses and their spectra for spin-orbit coupling cases 1 and 2. Compared to Fig. 2 where the spin-orbit coupling in the excited state was completely neglected, the optimal spectra of Fig. 6] are broader, with additional spectral amplitude at small and large frequency components. However, as explained in Section III, further calculations employing spectral filtering are necessary to determine whether these spectral features are artifacts of the optimization algorithm or whether they represent a true physical requirement that the optimal pulse has to fulfill. As seen in Fig. 6] the minimum optimization time to yield a population trans- 
case 1
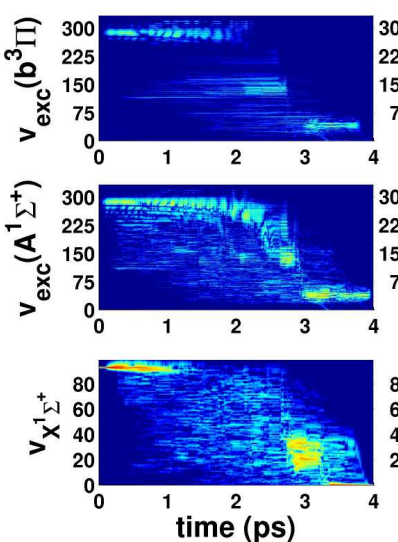

time (ps) case 2
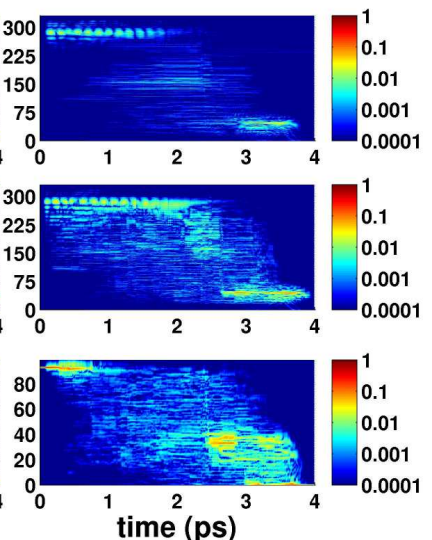

FIG. 7: (color online) Projection of the time-dependent wave packets onto the vibrational eigenstates of the electronic ground (bottom) and the coupled excited (top) states for spinorbit coupling cases 1 (left) and 2 (right).
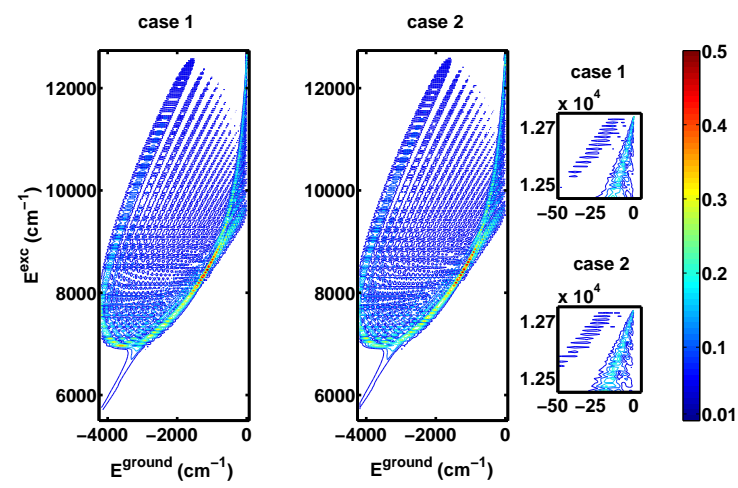

FIG. 8: (color online) Franck-Condon factors of the ground state levels with the singlet component of the excited state levels for $\mathrm{Na}_{2}$ (left) and KRb (right).

fer of better than $99 \%$ is not affected by the spin-orbit coupling in the excited state. This is in accordance with the rationalization in terms of the time scale of the vibrational dynamics on the electronic ground state, i.e. in terms of the timescales related to resolving the initial state and the target state, cf. Section III] The mininum required pulse energy amounts to $140 \mu \mathrm{J}$ for spin-orbit coupling case 1 and $180 \mu \mathrm{J}$ for case 2 . While the optimization target, i.e. population transfer of better than $99 \%$, can be achieved in both cases, case 2 which had been identified as potentially bad for the stabilization, requires more pulse energy. Both cases require substantially more pulse energy than the estimate of $61 \mu \mathrm{J}$ obtained with the two-state model of Section III. This is most likely due to the much larger state space that is explored by the optimization.

The dynamics under the optimal pulses are analyzed in Fig. 7 by projecting the time-dependent wave packet onto the triplet and singlet components of the vibrational eigenstates. Overall the dynamics are very similar for the two spin-orbit coupling cases. A difference would be expected mainly at the beginning of the pulse where the initial state is excited into levels of about $12630 \mathrm{~cm}^{-1}$ energy of the electronically excited state. Inspection of the Franck-Condon map displayed in Fig. 8 (inset) shows that the resonant coupling leads to additional peaks compared to the model without spin-orbit interaction. These features are caused by the additional peak in the singlet component of the vibrational eigenfunctions near the outer turning point of the triplet potential, cf. Fig. 5 . However, the projections of the wave packet within the first 1 ps do not reveal any substantial differences between coupling case 1 and 2 . We therefore conclude that the modifications of the Franck-Condon map due to the spinorbit interaction are not significant enough to influence the optimized stabilization dynamics, no matter whether the type of coupling is potentially favorable or potentially detrimental. The complete stabilization dynamics is rationalized in terms of the Franck-Condon map analogously to Section III, i.e. it is determined by the main ridges of the Franck-Condon map. The only difference between spin-orbit coupling cases 1 and 2 that can clearly be identified is the spread of population over the vibrational levels which is larger for case 2 .

To summarize the investigation of the influence of the spin-orbit interaction in the electronically excited state, the minimum optimization time is not affected while the required pulse energy is significantly increased compared to the model without spin-orbit interaction. Details of the spin-orbit interaction have only a minor effect on the required pulse energy and stabilization dynamics.

\section{OPTIMIZING TRANSFER FROM A SINGLET-TRIPLET SUPERPOSITION TO THE SINGLET GROUND STATE}

In heavy heteronuclear alkali dimer molecules it is possible to transfer a vibrationally excited state that is in the lowest triplet state or in a superposition of the lowest triplet and singlet electronic ground state, to the rovibronic ground state [8]. In order to study this as an optimization problem, a four-state model of the KRb molecule is considered, 


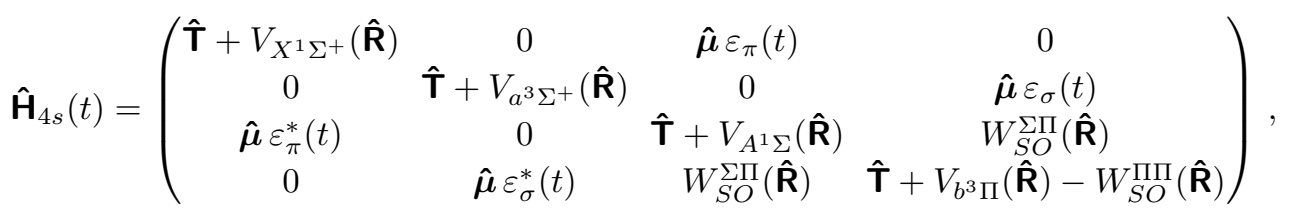

that allows for transfer of molecules from the lowest triplet state to the singlet ground state due to the spinorbit interaction in the electronically excited state. The lowest triplet state potential is taken from Ref. 37], the other potential curves, dipole moments and spin-orbit coupling functions are constructed as described in the previous sections. In particular, the spin-orbit coupling cases 1 and 2 introduced in Section [V] are employed. The initial state is taken to be a superposition of the vibrational eigenfunctions of the $a^{3} \Sigma^{+}$lowest triplet state and $X^{1} \Sigma^{+}$singlet electronic ground state with $4.5 \mathrm{~cm}^{-1}$ binding energy. The triplet (singlet) component carries $70 \%(30 \%)$ of the population. The target state remains unchanged compared to the previous sections, i.e. the $v=0$ level of the $X^{1} \Sigma^{+}$singlet electronic ground state.

Different laser polarizations need to be taken into account - linearly polarized light, $\varepsilon_{\pi}(t)$, for the singlet transitions and circularly polarized light, $\varepsilon_{\sigma}(t)$, for the triplet transitions. This simply means that instead of Eq. (6) two equations for the two components of the field need to be considered where the dot products are evaluated for the corresponding components of the states.

As explained above, a three-step optimization procedure is carried out in order to determine the minimum optimization time and minimum pulse energies. Also for the four-state model, population transfer with an efficiency of better than $99 \%$ is achieved by the optimal pulses. While the optimization time remains unchanged at $4 \mathrm{ps}$, the required pulse energy is increased. It amounts to $270 \mu \mathrm{J}$ for $\pi$-polarization and $230 \mu \mathrm{J}$ for $\sigma$-polarization in spinorbit coupling case 1 , and to $300 \mu \mathrm{J}$ for $\pi$-polarization and $270 \mu \mathrm{J}$ for $\sigma$-polarization in case 2 . As in Section IV. case 1 which is potentially favorable for the stabilization requires slightly less pulse energy than case 2 . However, the further increase of pulse energy compared to the three-state model of Section IV where pulse energies of $140 \mu \mathrm{J}$ and $180 \mu \mathrm{J}$ were obtained, is significant, in particular in view of the fact that the molecule now couples to two polarization components. The high pulse energies reflect the more difficult optimization problem that is considered here where the wave function needs to be changed qualitatively from a singlet-triplet superposition to a pure singlet state.

The dynamics under the optimal pulses are analyzed in terms of the projections of the time-dependent wave packet onto the components of the eigenfunctions on the four electronic states in Fig. 9. The dynamics of the singlet components is rather similar to those of the previous sections, cf. Figs. 3 and 7. Most of the triplet compo-

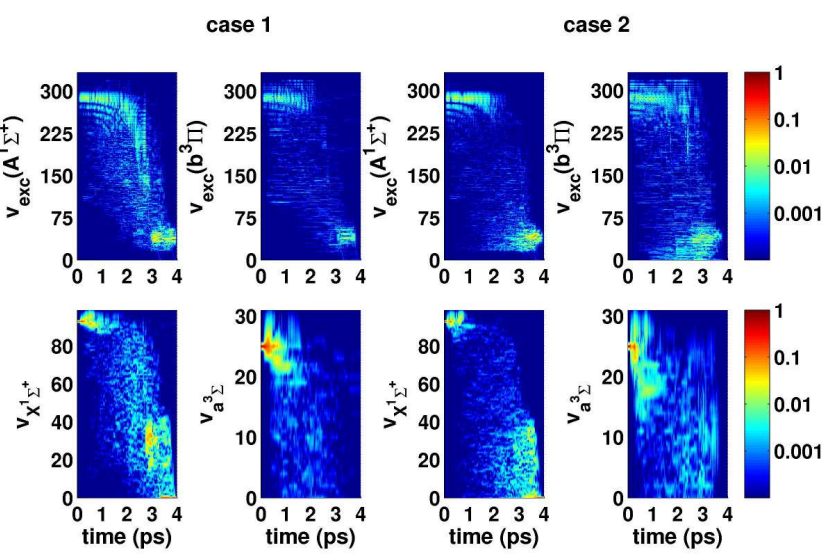

FIG. 9: (color online) Projection of the time-dependent wave packets onto the vibrational eigenstates of the electronic ground (bottom) and the coupled excited (top) states for spinorbit coupling cases 1 (left) and 2 (right).

nent of the initial state is converted to singlet components within the first half of the pulse, in particular in spin-orbit coupling case 1 . In the potentially detrimental case 2, more population resides in the triplet components than in case 1 and remains there throughout the pulse. The population dynamics should be compared to the Franck-Condon maps shown in Fig. 10. The singlet dynamics start out in the upper right corner of the Franck-Condon maps. They follow the main ridges of the map but fan out as the dynamics roll down the ridge and levels with equally likele transitions to many levels are populated. The triplet dynamics follows the right-most ridge in the upper right corner of the Franck-Condon maps. It never jumps over to the left-most ridge; and in particular in case 1 , the range between $v^{\prime}=275$ and $v^{\prime}=50$ of the $b^{3} \Pi$ state seems to be bridged via singlet dynamics. This is an indication for cooperative behavior between pulse and spin-orbit coupling in order to achieve the triplet-singlet transfer required by the optimization task: The dynamics travel down the right-most ridge of the Franck-Condon map due to Rabi cycling, i.e. due to interaction with the circularly polarized pulse. Then the spin-orbit coupling transfers most of the triplet population to the singlet channel, and a further decrease of the vibrational excitation happens in the singlet channels due to interaction with the $\pi$-polarized light. Such a cooperative behavior can be expected since the optimization 
case 1
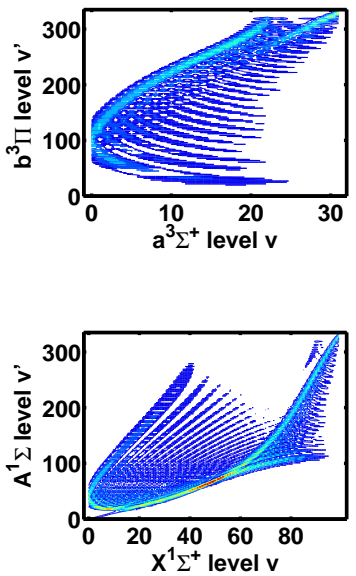

case 2
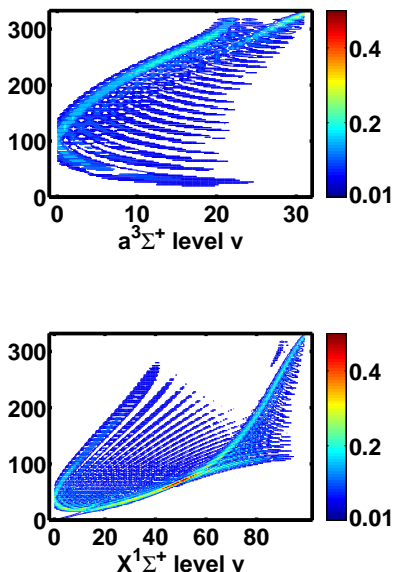

FIG. 10: (color online) Franck-Condon factors of the singlet $X$ ground state levels with the singlet component of the excited state levels and of the triplet $a$ ground state levels with triplet component of the excited state levels for spin-orbit coupling cases 1 (left) and 2 (right).

time is much larger than the time scale corresponding to the spin-orbit interaction. In the potentially detrimental case 2, no indication for cooperative behavior between pulse and singlet-triplet oscillations is observed; and population that was initially in the triplet channels remains there for a much longer time.

To summarize this section, vibrational stabilization from a singlet-triplet superposition to a pure singlet state can be achieved with better than $99 \%$ efficiency. However, even more pulse energy for both polarization components is required compared to stabilization of a pure singlet state. Depending on the details of the spin-orbit coupling function, cooperative behavior between pulse and spin-orbit coupling may or may not be observed.

\section{CONCLUSIONS}

We have studied the vibrational transfer of $\mathrm{KRb}$ molecules from a level just below the dissociation limit to the vibrational ground state. Optimal control theory was employed to obtain the shaped laser pulses that drive this population transfer with an efficiency of $99 \%$ or better. As the main result, our calculations have yielded an estimate of the minimum time that is required for the vibrational transfer, i.e. the quantum speed limit for this process [46], and an estimate on the required pulse energy.

Our findings have confirmed that optimal control approaches work as 'black-box' algorithms that provide solutions independent of the details of a given quantum object. Nevertheless, our results cannot straightforwardly be transferred to a laser pulse shaping experiment on cold molecules. Insufficient knowledge of the molecular structure and restriction of our model to a few electronic states prevent direct experimental application of the calculated pulses. This is a common phenomenon encountered in the optimal control of complex quantum systems such as molecules 47] as opposed to atoms [48] or spin systems [49]. In principle, our model could be refined. For example, Ref. [12] discusses in detail how potential loss channels such as multiphoton ionization could be incorporated; and more spectroscopy could be performed to obtained a better knowledge of the potential curves and non-adiabatic couplings. However, such refinement would miss the point of this study. Here, we come to a two-fold conclusion: On one hand, our study encourages optimal control experiments because solutions will be found no matter what are the specific details of the molecule. On the other hand, our study has clarified, as discussed below, the influence of the molecular structure relevant for the vibrational transfer by successively increasing the complexity of the model. While of less importance in optimal control experiments based on feedback loops, these findings are important for vibrational transfer and vibrational cooling using cw lasers, incoherent broadband light or adiabatic passage.

First, we have addressed the role of the long-range behavior of the excited state potential on the vibrational stabiliziation by comparing the $\mathrm{KRb}$ and $\mathrm{Na}_{2}$ molecules. From a time-dependent perspective one might expect the $1 / R^{3}$ potential of the homonuclear sodium dimer to be more favorable for the vibrational transfer since the larger slope of the potential speeds up the motion toward shorter internuclear distances. However, from a time-independent perspective, one might argue that the $1 / R^{6}$ excited state potential of heteronuclear molecules yields better Franck-Condon overlap with the electronic ground state that also shows a $1 / R^{6}$ dependence at large internuclear distances. Comparing a two-state model, i.e. a model comprising of the singlet electronic ground state and a single excited state, for $\mathrm{Na}_{2}$ and $\mathrm{KRb}$, we found that significantly less pulse energy is required for $\mathrm{KRb}$. We therefore conclude that a $1 / R^{6}$ excited state potential is more favorable for vibrational transfer than a $1 / R^{3}$ potential, i.e. the spectroscopic perspective prevails over the dynamical one.

The comparison of the $\mathrm{KRb}$ and $\mathrm{Na}_{2}$ molecules has also allowed us to identify what determines the minimum optimization time, i.e. the quantum speed limit for vibrational stabilization. The longest timescale in the problem that needs to be resolved by the optimal pulse is the vibrational motion of the initial state. Taking comparable binding energies of the initial state for $\mathrm{KRb}$ and $\mathrm{Na}_{2}$, the difference in the vibrational periods is due to the mass of the molecules, and the minimum optimization time is smaller for the lighter molecule.

Second, we have increased the complexity of the model for the molecular structure by taking spin-orbit coupling in the electronically excited state into account. The resulting model consists of three electronic states where the two electronically excited states exhibit a non-adiabatic coupling. In heavy alkali dimer molecules, the spin- 
orbit interaction does not only modify the potentials at large internuclear separation, it may also cause a mixing of vibrational ladders affecting the complete vibrational spectrum. This effect has been termed 'resonant coupling, 21 23]. Since the $R$-dependence of the spinorbit coupling function for KRb is not precisely known, we have adapted a parametrization developed by Bergeman et al. for RbCs 44]. In order to see which effect resonant spin-orbit coupling may have on the stabilization dynamics, we have modified the parameters of the coupling yielding a potentially favorable and a potentially detrimental case. While the resulting parametrization may be far from the true spin-orbit coupling found in the $\mathrm{KRb}$ molecule, this approach allows us to identify the maximum influence that the spin-orbit coupling may have on the stabilization dynamics. To our surprise, we found that in both the potentially favorable and the potentially detrimental coupling case, the pulse energy required to achieve population transfer to better than $99 \%$ is significantly increased compared to the two-state model neglecting the spin-orbit interaction. This means that the increased size of the state space has a much larger effect on the optimization than a modification of the Franck-Condon factors underlying the dynamics. If compared amongst each other, the potentially detrimental spin-orbit coupling case requires more pulse energy than the potentially favorable one. Overall, however, the details of the spin-orbit interaction seem to have only a minor effect on the stabilization dynamics.

Third, the spin-orbit interaction in the electronically excited state allows for population transfer from a singlettriplet superposition to a pure singlet level. In order to investigate this as an optimization problem, our minimal model consists of four electronic states, the singlet ground and lowest triplet state and the non-adiabatically coupled electronically excited states. Due to the symmetry of the electronic states, different polarization components of the laser field couple to transitions between the singlet and triplet channels. We found that the further increase in size of the state space as compared to the three-channel model results in even higher required pulse energies to achieve population transfer of $99 \%$ or better. For a shape of the spin-orbit coupling function that is potentially favorable to the vibrational transfer, we have observed indication for cooperative behavior between the pulse and the singlet-triplet transfer due to spin-orbit coupling. We have not seen any evidence for cooperative behavior in the case of potentially detrimental spin-orbit coupling. Correspondingly, the required pulse energy is larger for the potentially detrimental spin-orbit coupling case. Since the triplet-singlet transfer is explicitly part of the optimization problem in the four-state model, it is not surprising that details of the spin-orbit interaction play a somewhat larger role than for the three-state model for singlet-to-singlet vibrational population transfer.

In summary, independently of the details of the molecular structure, we have found optimal pulses achieving vibrational population transfer of KRb molecules to the vi- brational ground state with $99 \%$ efficiency or better. This highlights the power of the optimal control approach. However, as the complexity of the molecular structure is increased, the optimal laser fields need to carry more and more pulse energy. Each individual solution does of course depend on the details of the molecular structure, and we have analyzed the dynamics under the optimal pulse in terms of the underlying Franck-Condon maps.

In the present paper, the initial state was taken to be a highly excited but pure state. Such a situation is encountered for example if the molecules are created utilizing a Feshbach resonance [17]. Transfer to the vibrational ground state can then be achieved in a purely coherent process where the pulse absorbs the vibrational excitation energy of the molecule. The optimization task becomes more involved if the initial state corresponds to an incoherent ensemble of vibrationally excited molecules. Such a situation occurs if the molecules are created by photoassociation followed by spontaneous emission [3]. A true cooling scheme is then required where the molecules can dispose of energy and entropy [10]. While the present study was confined to vibrational stabilization, it may nevertheless shed light on the prospects for vibrational cooling. The Franck-Condon map shows a characteristic parabola whose distribution of weights and tilt determine whether the probability for heating is larger than that for cooling or vice versa. Similarly to the case of LiCs discussed in Ref. [50], the Franck-Condon maps presented above for $\mathrm{Na}_{2}$ and $\mathrm{KRb}$ reveal that vibrational cooling by optical pumping with a spectrally cut femtosecond laser pulse [10] will not be successful. In order to preferentially cool instead of heat despite the Franck-Condon map, a more sophisticated approach than optical pumping would be required. Here again, optimal control can serve as the tool of choice. For a toy molecular model, optimally shaped laser pulses together with spontaneous emission have been predicted to yield a successful cooling scheme [51].

Finally, we point out that we do not obtain an adiabatic passage-like solution since this is not accessible within our current optimization approach [52]. In fact, Stimulated Raman Adiabatic passage and related solutions formally require the infinite time limit [53]; and a finite optimization time needs to be fixed when numerically solving the optimization equations. So on one hand optimal control is an extremely convenient tool that allows for solving very complex optimization problems. On the other hand, however, it is not always straightforward to translate physical considerations such as allowing for the adiabatic limit into mathematical prescriptions for the algorithm. Our future work is therefore dedicated to developing more versatile optimization algorithms.

\section{Acknowledgments}

Fabian Borschel has contributed to this work at its initial stage. Financial support from the Deutsche 
Forschungsgemeinschaft is gratefully acknowledged.

[1] R. Krems, W. Stwalley, and B. Friedrich, eds., Cold Molecules. Theory, Experiment, Applications (CRC Press, 2009).

[2] S. Y. van de Meerakker, N. Vanhaecke, and G. Meijer, Annu. Rev. Phys. Chem. 57, 159 (2006).

[3] F. Masnou-Seeuws and P. Pillet, Adv. in At., Mol. and Opt. Phys. 47, 53 (2001).

[4] K. M. Jones, E. Tiesinga, P. D. Lett, and P. S. Julienne, Rev. Mod. Phys. 78, 483 (2006).

[5] T. Köhler, K. Góral, and P. S. Julienne, Rev. Mod. Phys. 78, 1311 (pages 51) (2006).

[6] K.-K. Ni, S. Ospelkaus, M. H. G. de Miranda, A. Pe'er, B. Neyenhuis, J. J. Zirbel, S. Kotochigova, P. S. Julienne, D. S. Jin, and J. Ye, Science 322, 231 (2008).

[7] F. Lang, K. Winkler, C. Strauss, R. Grimm, and J. H. Denschlag, Phys. Rev. Lett. 101, 133005 (2008).

[8] J. M. Sage, S. Sainis, T. Bergeman, and D. DeMille, Phys. Rev. Lett. 94, 203001 (2005).

[9] J. Deiglmayr, A. Grochola, M. Repp, K. Mörtlbauer, C. Glück, J. Lange, O. Dulieu, R. Wester, and M. Weidemüller, Phys. Rev. Lett. 101, 133004 (2008).

[10] M. Viteau, A. Chotia, M. Allegrini, N. Bouloufa, O. Dulieu, D. Comparat, and P. Pillet, Science 321, 232 (2008).

[11] S. Ospelkaus, K.-K. Ni, G. Quéméner, B. Neyenhuis, D. Wang, M. H. G. de Miranda, J. L. Bohn, J. Ye, and D. S. Jin, Phys. Rev. Lett. 104, 030402 (2010).

[12] C. P. Koch, J. P. Palao, R. Kosloff, and F. MasnouSeeuws, Phys. Rev. A 70, 013402 (2004).

[13] G. Roati, F. Riboli, G. Modugno, and M. Inguscio, Phys. Rev. Lett. 89, 150403 (2002).

[14] G. Thalhammer, G. Barontini, L. De Sarlo, J. Catani, F. Minardi, and M. Inguscio, Phys. Rev. Lett. 100, 210402 (2008).

[15] D. Wang, J. Qi, M. F. Stone, O. Nikolayeva, H. Wang, B. Hattaway, S. D. Gensemer, P. L. Gould, E. E. Eyler, and W. C. Stwalley, Phys. Rev. Lett. 93, 243005 (2004).

[16] M. W. Mancini, G. D. Telles, A. R. L. Caires, V. S. Bagnato, and L. G. Marcassa, Phys. Rev. Lett. 92, 133203 (2004).

[17] C. Ospelkaus, S. Ospelkaus, L. Humbert, P. Ernst, K. Sengstock, and K. Bongs, Phys. Rev. Lett. 97, 120402 (2006).

[18] C. Klempt, T. Henninger, O. Topic, M. Scherer, L. Kattner, E. Tiemann, W. Ertmer, and J. J. Arlt, Phys. Rev. A 78, 061602 (2008).

[19] K. Aikawa, D. Akamatsu, J. Kobayashi, M. Ueda, T. Kishimoto, and S. Inouye, New J. Phys. 11, 055035 (2009).

[20] J. T. Kim, D. Wang, E. E. Eyler, P. L. Gould, and W. C. Stwalley, New J. Phys. 11, 055020 (2009).

[21] C. Amiot, O. Dulieu, and J. Vergès, Phys. Rev. Lett. 83, 2316 (1999).

[22] H. K. Pechkis, D. Wang, Y. Huang, E. E. Eyler, P. L. Gould, W. C. Stwalley, and C. P. Koch, Phys. Rev. A 76, 022504 (2007).

[23] S. Ghosal, R. J. Doyle, C. P. Koch, and J. M. Hutson, New J. Phys. 11, 055011 (2009).
[24] C. P. Koch and R. Kosloff, Phys. Rev. Lett. 103, 260401 (2009), arXiv:0905.3251.

[25] P. Naidon and F. Masnou-Seeuws, Phys. Rev. A 68, 033612 (2003).

[26] R. Kosloff, Annu. Rev. Phys. Chem. 45, 145 (1994).

[27] V. Kokoouline, O. Dulieu, R. Kosloff, and F. MasnouSeeuws, J. Chem. Phys. 110, 9865 (1999).

[28] K. Willner, O. Dulieu, and F. Masnou-Seeuws, J. Chem. Phys. 120, 548 (2004).

[29] S. Kallush and R. Kosloff, Chem. Phys. Lett. 433, 221 (2006).

[30] J. P. Palao and R. Kosloff, Phys. Rev. A 68, 062308 (2003).

[31] V. F. Krotov, Doklady Mathematics 78, 949 (2008).

[32] V. F. Krotov, Automation and Remote Control 70, 357 (2009).

[33] S. E. Sklarz and D. J. Tannor, Phys. Rev. A 66, 053619 (2002).

[34] A. I. Konnov and V. F. Krotov, Automation and Remote Control 60, 1427 (1999)

[35] C. Samuelis, E. Tiesinga, T. Laue, M. Elbs, H. Knöckel, and E. Tiemann, Phys. Rev. A 63, 012710 (2000).

[36] E. Tiemann, H. Köckel, and H. Richling, Z. Phys. D. 37, 323 (1996).

[37] A. Pashov, O. Docenko, M. Tamanis, R. Ferber, H. Knöckel, and E. Tiemann, Phys. Rev. A 76, 022511 (2007).

[38] S. Rousseau, A. Allouche, and M. Aubert-Frcon, Journal of Mol. Spectrosc. 203, 235 (2000), ISSN 0022-2852.

[39] M. Marinescu and H. R. Sadeghpour, Phys. Rev. A 59, 390 (1999).

[40] S. Kallush and R. Kosloff, Phys. Rev. A 76, 053408 (2007).

[41] M. Lapert, R. Tehini, G. Turinici, and D. Sugny, Phys. Rev. A 79, 063411 (2009).

[42] M. Schröder and A. Brown, New J. Phys. 11, 105031 (13pp) (2009).

[43] T. Bergeman, J. Qi, D. Wang, Y. Huang, H. K. Pechkis, E. E. Eyler, P. L. Gould, W. C. Stwalley, R. A. Cline, J. D. Miller, et al., J. Phys. B 39, S813 (2006).

[44] T. Bergeman, C. E. Fellows, R. F. Gutterres, and C. Amiot, Phys. Rev. A 67, 050501 (2003).

[45] C. P. Koch, R. Kosloff, and F. Masnou-Seeuws, Phys. Rev. A 73, 043409 (2006).

[46] T. Caneva, M. Murphy, T. Calarco, R. Fazio, S. Montangero, V. Giovannetti, and G. E. Santoro, Phys. Rev. Lett. 103, 240501 (2009).

[47] T. Hornung, M. Motzkus, and R. de Vivie-Riedle, J. Chem. Phys. 115, 3105 (2001)

[48] T. Baier, M. Wollenhaupt, C. Sarpe-Tudoran, and T. Baumert, Phys. Rev. Lett. 102, 023004 (2009).

[49] T. E. Skinner, T. Reiss, B. Luy, N. Khaneja, and S. J. Glaser, J. Magn. Reson. 163, 8 (2003).

[50] R. Kosloff, P. Pillet, C. P. Koch, J. Ye, P. Barker, J. Deiglmayr, and M. Pichler, Faraday Discuss. 142, 319 (2009).

[51] A. Bartana, R. Kosloff, and D. J. Tannor, Chem. Phys. 267, 195 (2001). 
[52] Y. B. Band and O. Magnes, J. Chem. Phys. 101, 7528 (1994).

[53] H. Yuan, C. P. Koch, P. Salamon, and D. J. Tannor, arXiv:1004.4050v1 (2010).

[54] Please note that an incorrect value for the 3S-3P exci- tation energy of sodium was considered in Ref. [12]. All spectra need to be shifted by $5991 \mathrm{~cm}-1$. The conclusions of Ref. 12] with respect to spectral bandwidth, required optimization time and pulse energy remain unchanged. 